\title{
Minimally invasive open nephrectomy on children with multicystic dysplastic kidney
}

\author{
DONGCHUAN FENG, XIAOYU ZHU, FANG SUN, TONGSHENG MA, YUAN LI and SHUJING CHEN
}

Department of Urology, Xuzhou Children's Hospital, Xuzhou, Jiangsu 221002, P.R. China

Received November 25, 2015; Accepted September 30, 2016

DOI: $10.3892 /$ etm.2016.3816

\begin{abstract}
The aim of the study was to summarize the preliminary experience of minimally invasive open nephrectomy operation on children with multicystic dysplastic kidney (MCDK). A retrospective review was performed on the clinical materials of the 15 children that had accepted consecutive minimally invasive open nephrectomies during the previous 2 years. The enrolled children were diagnosed with unilateral MCDK under computed tomography, emission computerized tomography and ultrasound and no anomaly in the contralateral functioning kidney was found. Of the 15 children, 12 were boys and 3 were girls, with 5 cases on the right and 10 cases on the left. Operations were completed at the retroperitoneal space in order to open an incision on the waists and ribs of the children, the length of which ranged from 1.5 to $2.0 \mathrm{~cm}$ (average $1.7 \mathrm{~cm}$ ). The age of the children at operation ranged from 3 months to 5.6 years old, with an average of 2.4 years old. Surgery lasted for 30-50 min, with an average of $34.6 \mathrm{~min}$. The estimated blood loss of each child was $<5 \mathrm{ml}$. After operation, prophylactic intravenous antibiotics were administered for 2-4 days to prevent infection. All of the operations proved very successful. Following surgery the children were hospitalized for 2-4 days for observation, with an average of 2.8 days. No complications occurred during the follow-up period. In conclusion, minimally invasive open nephrectomy is effective for children with MCDK. The procedure is superior with regard to operative time, cosmesis, and length of stay. It is a safe and effective treatment choice for patietns with MCDK and can be easily performed on children.
\end{abstract}

\section{Introduction}

Multicystic dysplastic kidney (MCDK) is a common congenital renal malformations in children (1-3). Histologically, the embryonic structures of the affected kidney were poorly

Correspondence to: Dr Xiaoyu Zhu, Department of Urology, Xuzhou Children's Hospital, 18 Sudibei Road, Xuzhou, Jiangsu 221002, P.R. China

E-mail: zhuxiaoyu0305@yeah.net

Key words: multicystic dysplastic kidney, nephrectomy, minimally invasive operation differentiated. The diagnostic standard of MCDK includes replacement of the affected kidney by cysts of different size, although the cysts were not connected with each other. Renal parenchyma was not found (4-7).

Currently, there are some disputes on the treatment of MCDK, whether the affected kidney would do harm to the children and whether an operation is needed to remove the affected kidney. Some scholars held that the long-term conservative observation on MCDK was relatively safe and complications associated with it, such as high blood pressure and malignant tumors were negligible. Additionally, it was deemed that there was no need to remove the affected kidney $(8,9)$. Most of the scholars and parents that supported conservative treatment concentrated on the potential risks, the costs and injuries of the surgery itself $(10,11)$. However, other investigators support surgical resection of the affected kidney. Chengru suggested that MCDK had potential malignancy tendency (4) and that nephrectomy should be conducted on patients with unilateral MCDK. Kiyak et al diagnosed and treated 90 cases of unilateral MCDK and found that 41 cases $(45.5 \%)$ had undergone nephrectomy due to high blood pressure, repeated urinary tract infection, and abdominal pains. Those authors held that high blood pressure was quite common in children with MCDK, and the high blood pressure could be mitigated (12). Their findings were consistent with those of other authors (13-15). Seeman et al identified dynamic blood pressure in $25 \mathrm{MCDK}$ children and found that the blood pressure of 5 cases (20\%) was above the 95th percentiles, and there were 2 cases that suffered from high blood pressure both during the day and night, while the other 3 cases suffered from high blood pressure solely during the night (16). Their findings showed that the influence of MCDK on patient children was underestimated. Children that showed no symptoms were usually ignored in clinic diagnosis. Particularly in the countries where medical costs were closely associated with the family economic situation, the children were often lost during follow-up. Many children received only the minimum follow-up while, in fact, they needed a lifelong planned follow-up. There is evidence to indicate that long-term follow-up plays an important role in treatment adjustment and prognosis of MCDK (17-19). Furthermore, underage children were unable to cooperate, making to measure their blood pressure and the result reliability was worse than that of adults. Long-term follow-up was thought to result in spiritual pressure and potential physical harms, and which would be more harmful remains to be determined. 
It remains uncertain whether the affected kidney would produce any danger in children with MCDK. Kidney resection was considered a safe and effective option (20). However, we contended that the optimal approach was providing more information with regard to conservative and surgical treatment to the parents aiming to inform them of the advantages and disadvantages of conservative and surgical treatment, thereby including them in the choice of therapy. In the present study, 15 children that had accepted minimally invasive open nephrectomy during the period of May, 2008 and October, 2010 were enrolled.

\section{Materials and methods}

General materials. All of the enrolled children (12 boys and 3 girls) were unilateral MCDK. Of the 15 cases, 5 cases were on the right and 10 cases on the left. At the time of operation, the patients were aged from 3 months to 5.6 years old, with an average of 2.4 years old. All of them conformed to the diagnostic standard of MCDK: the B ultrasound showed that the affected kidney was replaced by cysts of different size, and that the cysts were not connected with each other. Renal parenchyma was not identified. Radionuclide imaging showed that the affected kidney had no functions. No anomaly was found on the contralateral functioning kidneys. Seven cases were discovered in the prenatal ultrasound examination, 3 cases were discovered in the asymptomatic medical ultrasound after birth, 3 cases were discovered due to repeated abdominal pain, and 2 cases were discovered due to abdominal mass. One case was found with mild hydronephrosis on the contralateral kidney. The affected kidneys on all the patients had no significant ectopia.

Following admission, all of the patients underwent blood pressure measurement, respectively, in the morning, afternoon, and the first half of the night at least once and no significant high blood pressure was found. All of the children underwent a computed tomography (CT) examination [11 cases underwent CT urography (CTU) examination, while 4 cases underwent plain CT scam], emission CT (ECT) examination and ultrasonic testing prior to surgery and renal function was normal. The CT showed that the affected kidney lost its normal form and was replaced by cysts of different size, and affected kidney was not strengthened in CTU. ECT was applied to detect split renal function and showed that the affected kidney had no function. Ultrasonic testing showed that the morphological structure of the affected kidney disappeared, and became a multiple cystic density image. The longest diameter of the affected kidney was $2.3-8.0 \mathrm{~cm}$ (average $4.4 \mathrm{~cm}$ ).

Methods. Endorhachis, combined with trachea cannula, was applied for anesthesia. The children were placed in a supine position. Their hypochondrium on the affected side was elevated. The operation table was leaned towards the healthy side for $20^{\circ}$. All of the enrolled children accepted minimally invasive open nephrectomy. The specific operation method involved making a small transverse incision from the lumbocostal part on the affected side. The center of the incision was $1 \mathrm{~cm}$ below the anterior axillary line, and costal margin. A rule was used to measure out a $1.5-2.0 \mathrm{~cm}$ incision. Subsequently, an incision was made from the skin to the subcutaneous tissue, aponeurosis of the abdominal external oblique muscle, abdominal internal oblique muscle, and abdominal transverse

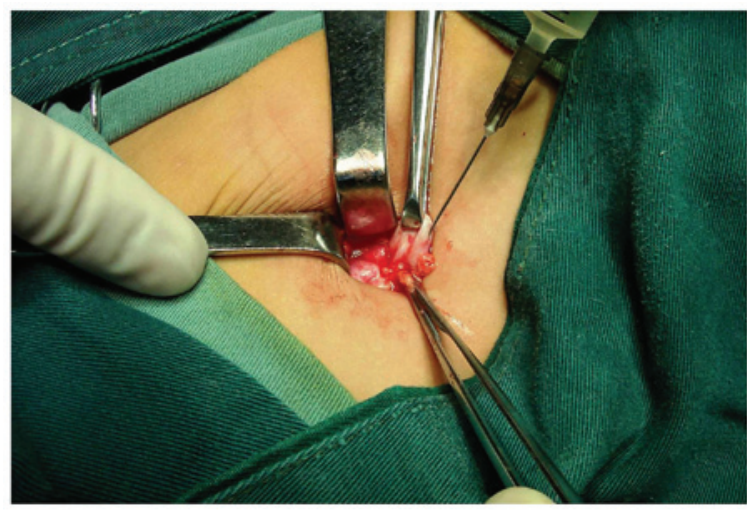

Figure 1. Syringe needle was connected to aspirator to extract vesicle fusion.

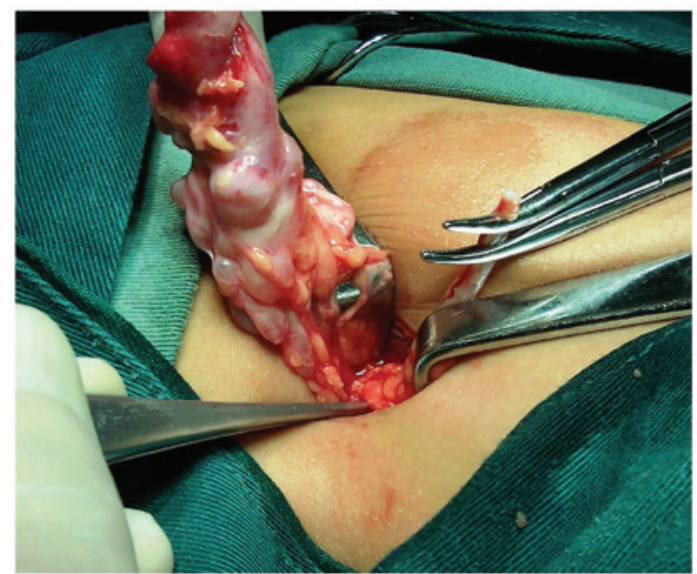

Figure 2. The affected kidney that was extracted via vesicle fusion was removed via incision.

muscle till peritoneum. The muscle, peritoneum, and perirenal fascia were carefully removed to expose the affected kidney. The affected kidney was removed using an allis clamp, while a no. 16 syringe needle with aspirator was simultaneously connected to extract vesicle fusion. Since vesica was not connected, each vesica that potentially blocked removal of the affected kidney was removed individually and the affected kidney was subsequently exposed (Figs. 1 and 2).

Renal pedicle vessels of the affected kidney were significantly maldeveloped and were handled by the combination of fulguration technique and ligation. After the affected kidney was exposed, the incision was pulled towards the direction of the pelvic cavity and ureter was fully dissociated towards the distal in order separate in a low position and conduct fulguration and ligation (Fig. 3). Following examination of the operating field, no active bleeding was identified. Subsequently, the muscle was sutured layer by layer.

Postoperative management. Following surgery, prophylactic intravenous antibiotics were administered for 2-4 days to prevent infection.

\section{Results}

Feasibility of the surgeries. All of the operations for a total of 15 children with MCDK (males, 12; females, 3) were 


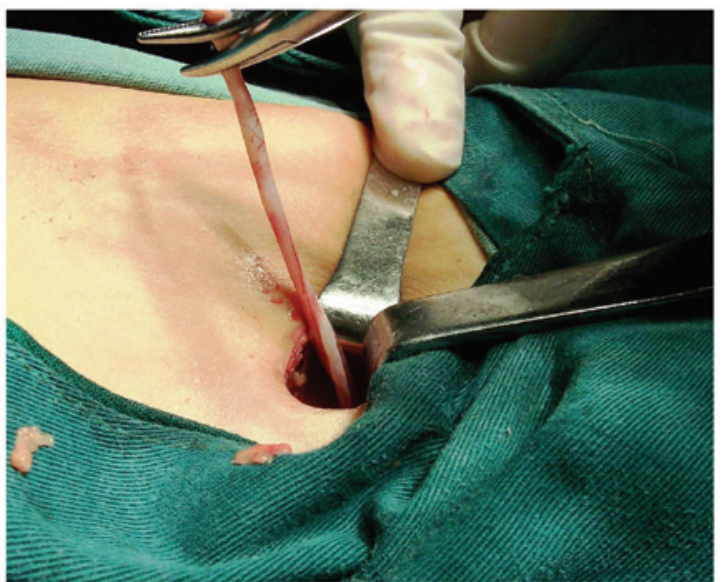

Figure 3. Dissociation of ureter towards the distal.

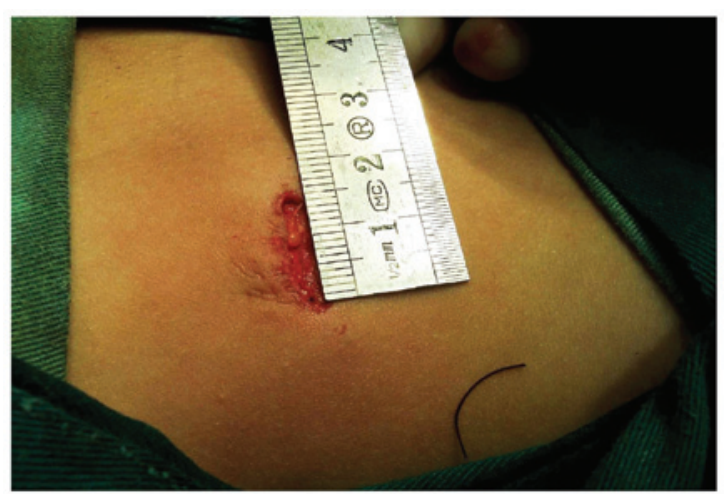

Figure 4. Measuring of the incision following surgery.

successfully performed via unilateral approach. The length of incision was 1.5-2.0 cm (average, $1.7 \mathrm{~cm}$ ) (Fig. 4). The age of the children at operation ranged from 3 months to 5.6 years old, with an average of 2.4 years old. Surgery lasted for 30-50 min, with an average of $34.6 \mathrm{~min}$ and estimated blood loss of each patient was $<5 \mathrm{ml}$. Pathology of all the enrolled cases conformed to MDCK.

Clinical outcomes. The average in-hospital time after operation was 2.8 days (range, 2-4 days). B ultrasonography was used to review the results after a follow-up period of 1 and 2 months, respectively, and the results showed that no residue was left on the affected kidney and no complications occurred after operation.

\section{Discussion}

At present, there are mainly two types of surgical methods: open operation and laparoscopic surgery. For both surgical methods, extraperitoneal approach was the best option. Hui et al (21) demonstrated that skillful retroperitoneal laparoscopic nephrectomy could accomplish the effects of open operation, and its surgical injuries and possibility for complications was much less than those of traditional open operation and laparoscopic surgery. However, it was difficult to handle in operation and required a longer operative time and learning curve. Additionally, retroperitoneal laparoscopic nephrectomy required creating a large retroperitoneal operation gap, which was difficult to achieve directly through trocar puncture.

Generally, it required a $2-\mathrm{cm}$ open incision. The retroperitoneal space was opened manually and a water balloon or a balloon was also needed to dilate enough operation space. Additionally, $\geq 3$ trocar channels were required to be established, leading to a large operation injury. Compared with minimally invasive open nephrectomy operation, laparoscopic surgery was deemed to have greater advantages. However, it was contended that open surgery that was conducted under the guidance of the concept of minimally invasive technique should be considered as the basis for comparative study (22-26). Introduction of minimally invasive surgery into the regular operation may significantly reduce operation injuries (23). At present, to the best of our knowledge, no final conclusion has been reached on the comprehensive evaluation of injuries and effects of minimally invasive open nephrectomy operation and laparoscopy nephrectomy. However, whether laparoscopy is likely to replace open surgery or not in the future remains to be determined.

In the present study, the operative incision was $1.5-2.0 \mathrm{~cm}$, thus far, no failed cases have been identified, thus proving that minimally invasive open invasion was feasible for the resection of the affected kidney of children (27). In order to conduct minimally incisive open surgery, attention was focused on (26): i) operation indication. This operation was applicable for preschool children with MCDK, whose disease was unilateral, and the longest diameter of whose affected kidney was $>2 \mathrm{~cm}$ without significant ectopia. However, whether this operation was applicable for the older children, whose longest diameter of the affected kidney was $<2 \mathrm{~cm}$ and who were also accompanied with pelvic cavity ectopia, required further clinical study. ii) Selection of the incision. A small transverse incision on the lumbocostal part on the affected side was selected and the center of the incision was $1 \mathrm{~cm}$ below the anterior axillary line, and costal margin. For the patients whose affected kidney was relatively small and who had mild ectopia, we applied B ultrasonography to locate the kidney and make a mark. The incision was located in the middle of the affected kidney. A steel rule was used to measure out a $1.5-2.0 \mathrm{~cm}$ incision that could lean towards the side of body. Although this potentially increased the difficulty of the operation, the cosmetic result thereof was better. iii) Precise choice of the admission passage. After the muscular layer was cut open, a small drag hook was used to pull the muscle open and the side peritoneum, which was exposed, was then pushed open from outside in. The kidney capsule was freed manually (usually, an incision of $2 \mathrm{~cm}$ supported the entry of the index finger and incision of $1.5 \mathrm{~cm}$ supported entry of the little finger). A knife or tweezer handle was also to obtain a smaller incision. After the kidney capsule was disconnected, the muscle was manipulated mannually to expose the affected kidney. Notably, the position of the kidney capsule was relatively deep; thus, we were required to open the peritoneum more deeply. In the case that the peritoneum was open up in error, it was sutured timely and the procedure was continued as locating the affected kidney was imperative for this operation. After the affected kidney was located, an allis clamp was used to clinch the affected kidney and lift it outward. A no. 16 syringe needle was simultaneously used for puncturation and extraction of the effusion. Generally, we did not need to evacuate the entire vesica, but only the vesica that 
prevented removal of the affected kidney in order to free the affected kidney. The affected kidney usually had a very clear dividing line with the surrounding tissues and it was easy to remove. The development of the patients' renal pedicle vessels was usually poor, requiring binding up of the residue end to prevent it from bleeding. The incision was pulled towards the distal to free the ureter until it reached the pelvic cavity. iv) In the case without significant errhysis, drainage was saved. v) It was suggested that this operation be combined with minimally incisive open pyeloplasty.

Surgical procedures of these two methods were basically similar (28). Additionally, minimally invasive open nephrectomy operation was easier than minimally incisive open pyeloplasty. Combining the two methods together may be useful in accelerating learning. The advantages of minimally invasive open nephrectomy operation include: i) little operative injury, and rapid recovery. Compared with conventional invasive open nephrectomy operation and the present laparoscopy nephrectomy, this operation required a relatively small space and few organizational structures that needed dissection. The total length of operative incision was smaller than that of the present laparoscopy nephrectomy and regular operation. The estimated blood loss was $<5 \mathrm{ml}$. Postoperative care was easy and convenient. Patients did not need to stay in bed for a long time. Postoperative recovery was very quick. Normal activities could be resumed on the second day after operation. Since the operative injury was small, parents of the patient children were more willing to select this operation. ii) Safe and reliable. All of the operations were completed under direct vision. No residue remained and renal pedicle vessels were managed safely. It was also easier to cut off the ureter. iii) The technique was relatively easier to master. The whole operation lasted for approximately $35 \mathrm{~min}$. iv) Low requirements on surgical instruments. Surgery was conducted using normal surgical instruments, which was conducive to the popularization of minimally invasive open nephrectomy operation. Our study assumed that minimally invasive open nephrectomy operation had some advantages over laparoscopy nephrectomy. Although the number of cases was relatively small and the method remains to be studied further, primary experience already indicates that minimally invasive open nephrectomy operation has advantages in terms of its minimal invasion, operability, and operation results.

\section{References}

1. Sharada S, Vijayakumar M, Nageswaran P, Ekambaram S and Udani A: Multicystic dysplastic kidney: a retrospective study. Indian Pediatr 51: 641-643, 2014.

2. Haija MA, Qian YW and Muthukumar A: Dyserythropoiesis in a child with pyruvate kinase deficiency and coexistent unilateral multicystic dysplastic kidney. Pediatr Blood Cancer 61: $1463-1465,2014$

3. Guo Q, Tripathi P, Manson SR, Austin PF and Chen F: Transcriptional dysregulation in the ureteric bud causes multicystic dysplastic kidney by branching morphogenesis defect. J Urol 193 (Suppl 5): 1784-1790, 2015.

4. Bian H: Practical Pediatric Urology Surgery. People's Medical Publishing House, Beijing, p187, 2006.

5. Al Naimi A, Baumüller JE, Spahn S and Bahlmann F: Prenatal diagnosis of multicystic dysplastic kidney disease in the second trimester screening. Prenat Diagn 33: 726-731, 2013.
6. Tonni $\mathrm{G}$ and Grisolia G: Ultrasound diagnosis of central nervous system anomalies (bifid choroid plexus, ventriculomegaly, Dandy-Walker malformation) associated with multicystic dysplastic kidney disease in a trisomy 9 fetus: case report with literature review. J Clin Ultrasound 41: 441-447, 2013.

7. Canning DA: Re: Unilateral multicystic dysplastic kidney: does initial size matter? J Urol 190: 1893-1894, 2013.

8. Singh JK, Kanojia RP and Narasimhan KL: Multicystic dysplastic kidney in children - a need for conservative and long term approach. Indian J Pediatr 76: 809-812, 2009.

9. Mattioli G, Pini-Prato A, Costanzo S, Avanzini S, Rossi V, Basile A, Ghiggeri GM, Magnasco A, Leggio S, Rapuzzi G, et al: Nephrectomy for multicystic dysplastic kidney and renal hypodysplasia in children: Where do we stand? Pediatr Surg Int 26: 523-528, 2010.

10. Han JH, Lee YS, Kim MJ, Lee MJ, Im YJ, Kim SW and Han SW: Conservative management of segmental multicystic dysplastic kidney in children. Urology 86: 1013-1018, 2015.

11. Okada T, Yoshida H, Matsunaga T, Kouchi K, Ohtsuka Y, Saitou T, Matsuura G and Ohnuma N: Multicystic dysplastic kidney detected by prenatal ultrasonography: Natural history and conservative management. Pediatr Surg Int 19: 207-210, 2003.

12. Kiyak A, Yilmaz A, Turhan P, Sander S, Aydin G and Aydogan G: Unilateral multicystic dysplastic kidney: single-center experience. Pediatr Nephrol 24: 99-104, 2009.

13. Whittam BM, Calaway A, Szymanski KM, Carroll AE, Misseri R, Kaefer M, Rink RC, Karmazyn B and Cain MP: Ultrasound diagnosis of multicystic dysplastic kidney: is a confirmatory nuclear medicine scan necessary? J Pediatr Urol 10: 1059-1062, 2014

14. Xu J, Chen DP, Mao ZG, Huang HF, Xu CM, Wang CR, Jia WP and Mei CL: Autosomal dominant polycystic kidney disease with ectopic unilateral multicystic dysplastic kidney. BMC Nephrol 14: 38, 2013.

15. Kalisvaart J, Bootwala Y, Poonawala H, Elmore J, Kirsch A, Scherz H, Jones R, Grattan-Smith JD and Smith E: Comparison of ultrasound and magnetic resonance urography for evaluation of contralateral kidney in patients with multicystic dysplastic kidney disease. J Urol 186: 1059-1064, 2011.

16. Seeman T, John U, Bláhová K, Vondrichová H, Janda J and Misselwitz J: Ambulatory blood pressure monitoring in children with unilateral multicystic dysplastic kidney. Eur J Pediatr 160: 78-83, 2001.

17. Sarhan OM, Alghanbar M, Alsulaihim A, Alharbi B, Alotay A and Nakshabandi Z: Multicystic dysplastic kidney: Impact of imaging modality selection on the initial management and prognosis. J Pediatr Urol 10: 645-649, 2014.

18. Cooper CJ, Said S, Khalillullah S, Salameh HJ and Hernandez GT: Multicystic dysplastic kidney complicated by pyelonephritis. Am J Case Rep 14: 412-415, 2013.

19. Moralıoğlu S, Celayir AC, Bosnalı O, Pektaş OZ and Bulut IK: Single center experience in patients with unilateral multicystic dysplastic kidney. J Pediatr Urol 10: 763-768, 2014.

20. Nouira F, Sarrai N, Ghorbel S, Sghair YO, Khemakhem R, Chariag A, Jlidi S and Chaouachi B: Indications for nephrectomy in children: What has changed? Tunis Med 88: 253-256, 2010.

21. Hui C, Qi Y and Ying H: 9 cases of laparoscopic treatment on children with unilateral renal dysplasia. J Appl Clin Pediatr 25: 1361-1363, 2010.

22. Jiabo C, Tiquan Y and Yige L: Application of laparoscopic surgery on the treatment of diseases of the urogenital system in children. J Appl Clin Pediatr 25: 1598-1600, 2010.

23. Chacko JK, Koyle MA, Mingin GC and Furness PD 3rd: Minimally invasive open renal surgery. J Urol 178: 1575-1577; discussion 1577-1578, 2007.

24. Hayes WN and Watson AR; Trent \& Anglia MCDK Study Group: Unilateral multicystic dysplastic kidney: does initial size matter? Pediatr Nephrol 27: 1335-1340, 2012.

25. Eickmeyer AB, Casanova NF, He C, Smith EA, Wan J, Bloom DA and Dillman JR: The natural history of the multicystic dysplastic kidney - is limited follow-up warranted? J Pediatr Urol 10: 655-661, 2014.

26. Takeuchi M, Kamishima Y, Hara M, Horibe Y, Ishii M, Okumura K and Shibamoto Y: Segmental multicystic dysplastic kidney in an adult: Usefulness of enhanced CT in excretory phase. Abdom Imaging 38: 603-607, 2013.

27. Welch TR and Wacksman J: The changing approach to multicystic dysplastic kidney in children. J Pediatr 146: 723-725, 2005.

28. Dongchuan F, Xiaoyu Z and Hongwei Z: Minimal invasive open Anderson-Hynes operation. Chin J Pediatr Surg 31: 20-23, 2010. 